\title{
Transcriptional activation of PD-L1 by Sox 2 contributes to the proliferation of hepatocellular carcinoma cells
}

\author{
FENG ZHONG ${ }^{1}$, XINSHENG CHENG ${ }^{2}$, SHIBO SUN $^{3}$ and JIE ZHOU ${ }^{3}$ \\ ${ }^{1}$ Department of Hepatobiliary Surgery, Shenzhen Hospital, Southern Medical University, Shenzhen, \\ Guangdong 518100; ${ }^{2}$ Department of Hepatobiliary Surgery, Nanshan Hospital, Guangdong Medical College, \\ Shenzhen, Guangdong 518052; ${ }^{3}$ Department of Hepatobiliary Surgery, Nanfang Hospital, \\ Southern Medical University, Guangzhou, Guangdong 510515, P.R. China
}

Received September 27, 2016; Accepted March 3, 2017

DOI: $10.3892 /$ or.2017.5523

\begin{abstract}
Hepatocellular carcinoma (HCC) is one of the most common and lethal malignancies in the world. Sox 2 is a potential oncogene in the pathogenesis of HCC, however, the actual mechanisms of Sox 2 functions in HCC has not emerged yet. In this study, we explored the expression, function and the relationship between Sox 2 and PD-L1 in HCC. We found that both Sox 2 and PD-L1 were expressed at a markedly higher level in HCC tissues in comparison to adjacent non-tumor tissues. Moreover, the expression levels of both genes were correlated with each other. Knockdown of Sox 2 reduced the cell proliferation ability and induces apoptosis of HCC cells, suggesting the function of Sox 2 in regulating both the cell proliferation and apoptosis. Noteworthy, the depletion of Sox 2 also reduced the expression of PD-L1. Further analysis showed that there is a consensus Sox 2 binding site in the promoter region of PD-L1. Through in vitro EMSA assay and in vivo chromatin immunoprecipitation assays, we demonstrated that Sox 2 directly bound to the PD-L1 promoter through the consensus Sox 2 motif. Further evidence by luciferase reporter assays revealed that Sox 2 promoted the transcription activity of PD-L1 promoter region through the Sox 2 motif. Collectively, our data provide a novel insight into the function and the interplay of Sox 2 and PD-L1 in HCC.
\end{abstract}

\section{Introduction}

Hepatocellular carcinoma (HCC) is one of the most lethal malignant tumors in the world $(1,2)$. During the past decades, plenty of research regarding the genetic markers (i.e. abnormal gene expression as well as the genomic aberrations) have been

Correspondence to: Professor Jie Zhou, Department of Hepatobiliary Surgery, Nanfang Hospital, Southern Medical University, 1838 North Guangzhou Avenue, Guangzhou, Guangdong 510515, P.R. China

E-mail: zhoujkk@163.com; southzhoujie@163.com

Key words: hepatocellular carcinoma, Sox2, PD-L1 accumulated for HCC (3-7). The primary risk factors for the pathogenesis of HCC have been elucidated, and the multiple steps involved in hepatocarcinogenesis have been well defined in recent years (8). In spite of these associations between the risk factors and the development of HCC being wellestablished $(4,9)$, no clear picture of the actual mechanisms of how and what consequences these factors have leading to malignant transformation has emerged yet.

Sox 2 is a transcription factor that controls the expression of a number of target genes through forming a trimeric complex with Oct4 on DNA (10). Until now, the relationship between Sox 2 and tumorigenesis as well as overall survival (OS) has been well elucidated in several solid tumors, such as breast, esophageal and lung cancer (11-16). In addition, Sox2 has also been implicated in the progression of HCC. For instance, the expression levels of Sox 2 and its co-factor Oct 4 correlate with an aggressive phenotype and low survival rate of HCC (17). HCC cells overexpressing Sox 2 are characterized by increased ability of epithelial-mesenchymal transition (EMT), invasion and clonal formation (18). miR126, which is a tumor suppressor, inhibits cell growth through reducing the expression of Sox 2 (19). These studies indicate that Sox 2 functions as an oncogene in HCC. However, it is still unclear through which pathway Sox 2 promotes tumorigenesis.

PD-L1 (also known as B7-H1), a gene encoding an immune inhibitory receptor ligand, is expressed in various types of cancer and immune cells (i.e. B cells, T cells, and dendritic cells) (20-22). Binding of PD-L1 to its receptor PD-1 (the programmed cell death-1 receptor) leads to tumor evasion from host immune system (23), which is achieved through suppressing $\mathrm{T}$-cell response, migration, proliferation and restricting the tumor cell killing ability of T cells (21,24-26). Immunotherapeutics targeting the PD-L1/PD-1 pathway are currently in clinical trials and have shown impressive response rates in patients, particularly for non-small cell lung cancer (NSCLC), bladder cancer, renal cell carcinoma and melanoma $(27,28)$. It has been reported that the PD-L1 expression in HCC is significantly associated with tumor malignancy and the risk of postoperative recurrence. Therefore, PD-L1 might represent a potential target for HCC immunotherapy as well as a biomarker that aids in determining the prognosis both before and after therapy (29). 
In this study, we investigated the expression and transcriptional regulation of Sox 2 and PD-L1 in HCC. We report that the expression level of Sox 2 and PD-L1 is higher in HCC tissues and cell lines compared with adjacent nontumor tissues and normal liver cell lines. Furthermore, the expression of Sox 2 and PD-L1 in HCC tissues are positively correlated with each other. Functionally, we also demonstrate that knockdown of Sox 2 reduces the cell vitality, arrests the cell growth and induces the apoptosis of HCC cell lines. Moreover, Sox 2 regulates the expression of PD-L1 through directly binding to the Sox 2 consensus binding site on PD-L1 promoter region and regulating the promoter activity of PD-L1. This study describes the crucial role of Sox 2 in HCC, and for the first time we studied the relationship between Sox 2 and PD-L1.

\section{Materials and methods}

Cell culture. HL-7702, HepG2, SMMC7721, Huh7 and HEK293 cells were cultured in DMEM (Gibco, Carlsbad, CA, USA) containing $10 \%$ fetal bovine serum (Gibco).

$R N A$ extraction and reverse transcription-quantitive $P C R$ $(R T-q P C R)$. Total RNA of cell lines (HL-7702, HepG2, SMMC7721, and Huh7) and patient tissues (hepatocellular carcinoma tissue and adjacent non-tumor tissue) were extracted using RNeasy Mini kit (Qiagen, Valencia, CA, USA) according to the manufacturer's protocol. RNase-free DNase I (Invitrogen, Carlsbad, CA, USA) were used to remove genomic DNA. Reverse-transcription was carried out with the Superscript III Reverse Transcriptase (Invitrogen). QPCR assays were performed with the SYBE Premix Ex Taq ${ }^{\mathrm{TM}}$ (Takara Bio, Otsu, Japan) on ABI ViiA 7 Real-time PCR System (Applied Biosystems, Foster City, CA, USA). The relative expression level of Sox 2 and PD-L1 were calculated as $2^{-[\mathrm{Ct}(\mathrm{Gene})-\mathrm{Ct}(\mathrm{GAPDH})]}(30)$. Each assay was performed three times. The primers used for RT-qPCR are shown in Table I.

RNA interference (RNAi). Lipofectamine ${ }^{\mathrm{TM}} 2000$ (Invitrogen) was used for siRNA delivery. SMMC7721 and HepG2 cells $\left(2.5 \times 10^{5}\right)$ were seeded in 6-well plate one day before transfection. A total amount of 100 pmol siRNA were diluted with $250 \mu \mathrm{l}$ of DMEM without serum and mixed gently. A total amount of $5 \mu \mathrm{l}$ Lipofectamine 2000 were mixed with $250 \mu \mathrm{l}$ of DMEM and incubated for $5 \mathrm{~min}$ at room temperature. Then the diluted DNA and the diluted Lipofectamine 2000 were firstly mixed gently and incubated for $20 \mathrm{~min}$ at room temperature. After $20 \mathrm{~min}$ of incubation, $500 \mu \mathrm{l}$ of DMEM/Lipo/siRNA mix were added to each well containing cells, and mixed gently. The cells were then incubated in a $\mathrm{CO}_{2}$ incubator containing $5 \% \mathrm{CO}_{2}$ at $37^{\circ} \mathrm{C}$. Cells were collected after $72 \mathrm{~h}$ of transfection for mRNA and protein extraction. A Sox 2 specific siRNA sequence (siSox2: 5'-CGGCUCUGUAUUAUUUGAATTA-3') was used for RNA interference. A mismatch siRNA sequence (siNC: 5'-UUCUCCGAACGUGUCACGUTT-3') was used as negative control.

Cell proliferation assay. Cell proliferation was detected by the Cell Counting Kit-8 (CCK-8) (Dojindo Laboratories, Kumamoto, Japan) and BrdU (Roche, Mannheim, Germany)
Table I. Primer sequences used for mRNA expression level analysis through qPCR.

\begin{tabular}{lc} 
Name & \multicolumn{1}{c}{ Primers sequence (5'-3') } \\
\hline Sox2-RT-F: & TACAGCATGTCCTACTCGCAG \\
Sox2-RT-R: & GAGGAAGAGGTAACCACAGGG \\
PD-L1-RT-F: & TGGCATTTGCTGAACGCATTT \\
PD-L1-RT-R: & TGCAGCCAGGTCTAATTGTTTT \\
GAPDH-RT-F: & TGTTCGTCATGGGTGTGAAC \\
GAPDH-RT-R: & ATGGCATGGACTGTGGTCAT \\
\hline
\end{tabular}

assays. The cell viability of HepG2 and SMMC7721 cells was detected by the CCK-8 (Dojindo Laboratories, Kumamoto, Japan). Briefly, $10 \mu \mathrm{l}$ of CCK-8 reagent was added at the 24,48 and $72 \mathrm{~h}$ siRNA posttransfection. Then cells were incubated at $37^{\circ} \mathrm{C}$ for $4 \mathrm{~h}$, and the number of viable cells was evaluated through measuring absorbance at $450 \mathrm{~nm}$.

EdU staining. HepG2 and SMMC7721 cells transfected with siRNA were used for EdU assay with Cell-Light ${ }^{\mathrm{TM}}$ EdU kit (RiboBio, Guangzhou, China) according to the manufacturer's protocol. Images were captured using a Leica DMI6000B microscope (Leica Microsystems, Wetzlar, Germany).

Apoptosisanalysis.siRNA-transfected HepG2 and SMMC7721 were collected for apoptosis analysis. Annexin V-FITC and PI (BD Biosciences) were used for detection of apoptotic cells.

Electrophoretic mobility shift assay (EMSA). EMSA was performed using Lightshift Chemiluminescent EMSA kit (Pierce, Rockford, IL, USA) according to the manufacturer's instructions. Biotin-labeled double-stranded oligonucleotide (5'-TATGACACCATCGTCTGTCATC-3') containing the consensus Sox 2 motif was used as EMSA probe. An unlabeled double-stranded oligonucleotide was used as competitor probe. An unlabeled-mutated oligonucleotide (5'-TATGACACGTAC CACTGTCATC) was used as negative competitor probe. Nuclear protein was extracted from HepG2 cells. Anti-Sox2 antibody (\#5024S, Cell Signaling Technology, Inc., Danvers, MA, USA) was used to supershift the DNA-protein complex.

Chromatin immunoprecipitation-quantitative-PCR (ChIP$q P C R)$. ChIP was performed according to the manufacturer manual (Active Motif, Carlsbad, CA, USA) in HepG2 cells with the anti-Sox 2 antibody (\#5024S). The qPCR assay was carried out with the SYBE Premix Ex Taq (Takara Bio) on ABI ViiA 7 Real-time PCR System. Normal rabbit IgG was used as negative control. ChIP-qPCR assay was performed in triplicate. The primers used for ChIP-qPCR are as follows: PD-L1 (forChIP-qPCR)-F:AAGAAAAGGGAGCACACAGG, PD-L1 (for ChIP-qPCR)-R: GCCCAAGATGACAGACGATG.

Plasmid. The PD-L1 promoter region was PCR amplified from HepG2 cells and cloned into the pGL3-basic vector (PD-L1-WT). Mutation of the Sox 2 binding site on PD-L1-WT was performed by Quik Change Site Mutagenesis kit 
A
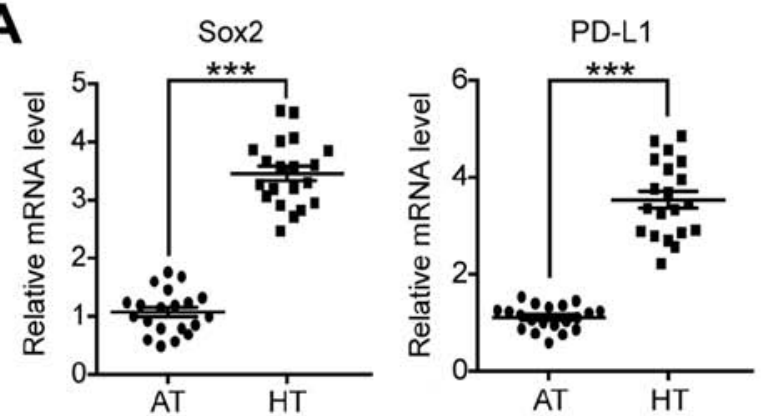

C

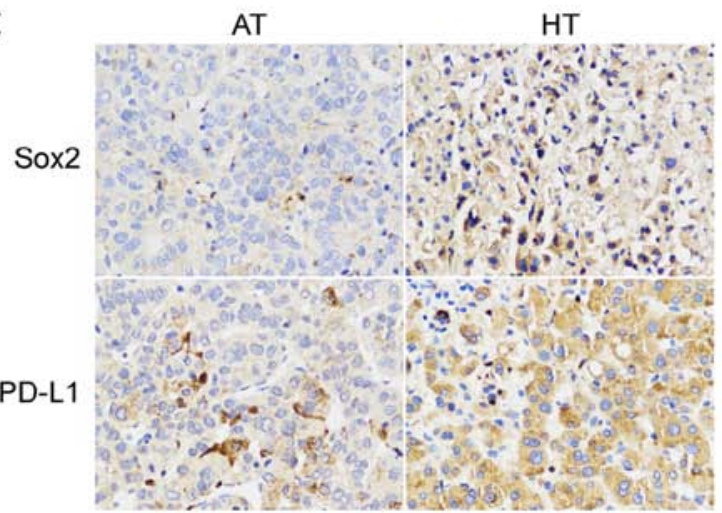

B

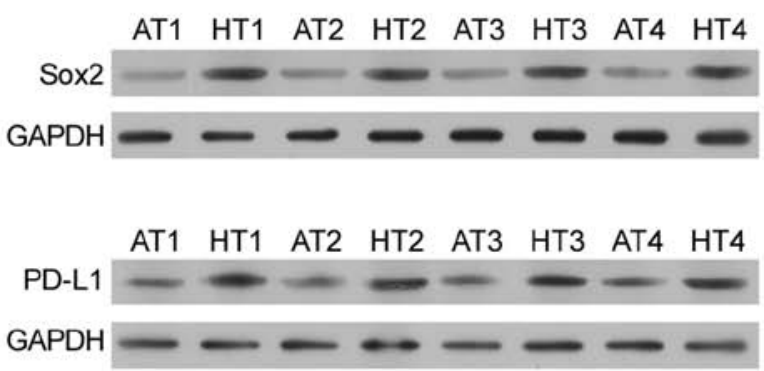

D

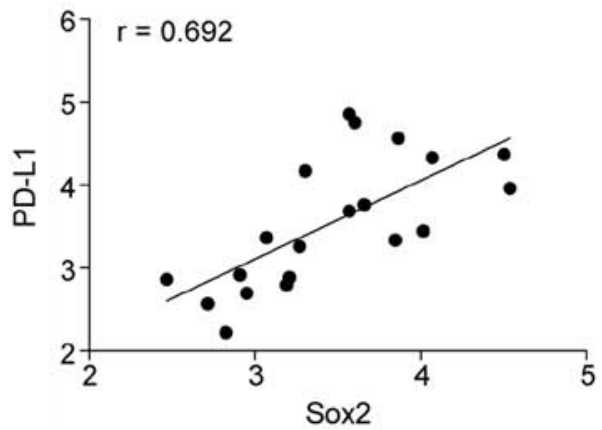

Figure 1. The expression of Sox 2 and PD-L1 expression are significantly higher in hepatocellular carcinoma (HCC) tissues adjacent to non-tumor tissues. (A) The mRNA level of Sox 2 (upper panel) and PD-L1 (lower panel) are significantly higher in HCC tissues than that in adjacent non-tumor tissues. (B) Western blotting shows that the protein level of Sox2 (upper panel) and PD-L1 (lower panel) are significantly higher in HCC tissues than that in adjacent non-tumor tissues. (C) Immunohistochemistry shows that the protein level of Sox 2 and PD-L1 are significantly higher in HCC tissues than that in adjacent non-tumor tissues. (D) Correlation analysis of Sox 2 and PD-L1 expression in HCC patients. AT, adjacent non-tumor tissue; HT, hepatocellular carcinoma tissue. Patient samples were retrieved from Department of Hepatobiliary Surgery, Shenzhen Hospital of Southern Medical University. ${ }^{* * *}$ p 0.001.

(Stratagene, La Jolla, CA, USA) according to the manufacturer's manual. Human Sox 2 cDNA was amplified from HepG2 cells by RT-PCR and cloned into the pSG5 vector. The primers used for cloning are as follows: PD-L1-Promoter-F: GGGGTA CCAGAAGGAAAGGCAAACAAC, PD-L1-Promoter-R: CCGCTCGAGCTTTGGGTTAGTGAATGGG; PD-L1Mutation-F: TACTTAAGTAAATTATGACATGCAGAC GTGTCATCTTGG, PD-L1-Mutation-R: CCAAGATGA CACGTCTGCATGTCATAATtTACTTAAGTA; Sox2cDNA-F: CGGGATCCTCTTCGCCTGATTTTCCTCG, Sox2-cDNA-R: GGAATTCCCTCCCATTTCCCTCGTTTT.

Luciferase. HEK293T cells were transfected using Lipofectamine 2000 (Invitrogen). Luciferase activity was detected by the Dual Luciferase Assay (Promega, Madison, WI, USA) after $24 \mathrm{~h}$ of transfection. Co-transfection of Renilla luciferase plasmid was used as the internal control for transfection efficiency.

Statistical analysis. Data in this research was presented as mean \pm SD, and t-test or one-way ANOVA was used among groups. $\mathrm{p}<0.05$ was considered statistically significant. Data were analyzed by using GraphPad Prism 5 for Windows (IBM, USA).

\section{Results}

Sox2 and PD-L1 expression are significantly higher in hepatocellular carcinoma (HCC) tissues than in adjacent non-tumor tissues. We initially compared Sox 2 and PD-L1 expression in HCC tissue samples and that in adjacent non- tumor tissue samples. We performed RT-qPCR and western blotting to measure Sox 2 and PD-L1 expression in HCC tissues versus adjacent non-tumor tissues from HCC patients. As illustrated in Fig. 1A and B, Sox 2 expression in HCC tissues (HT) was significantly higher than that in adjacent non-tumor tissues (AT) in both mRNA and protein levels. PD-L1 showed similar expression pattern as Sox2 (Fig. 1A, right panel, Fig. 1B, lower panel). To confirm this expression pattern, we conducted immunohistochemistry (IHC) staining to detect the Sox 2 and PD-L1 protein in the HCC tissue and adjacent non-tumor tissue samples. As compared with normal tissue, the Sox2 (Fig. 1C, upper panel) and PD-L1 (Fig. 1C, lower panel) signal is stronger in HCC tissue. Altogether, these analyses indicated that the expression of Sox 2 and PD-L1 might be correlated with each other. Thus, we further investigated the relationship between the expression level of Sox 2 and PD-L1. As shown in Fig. 1D, the expression level of Sox 2 was positively correlated with that of PD-L1 ( $r=0.692)$. Collectively, these results suggest that both Sox1 and PD-L1 are highly expressed in HCC tissue, and their expression are positive co-related with each other.

Sox2 and PD-L1 expression are significantly higher in HCC cell lines than that of normal liver cell line. We performed RT-qPCR to measure the mRNA level of Sox 2 and PD-L1 in HCC cell lines (Huh7, SMMC7721, and HepG2) and normal liver cell line (HL-7702). As shown in Fig. 2A, Sox 2 and PD-L1 expression in HCC cell lines were significantly higher than that in the normal liver cell line. We also analyzed the 
A

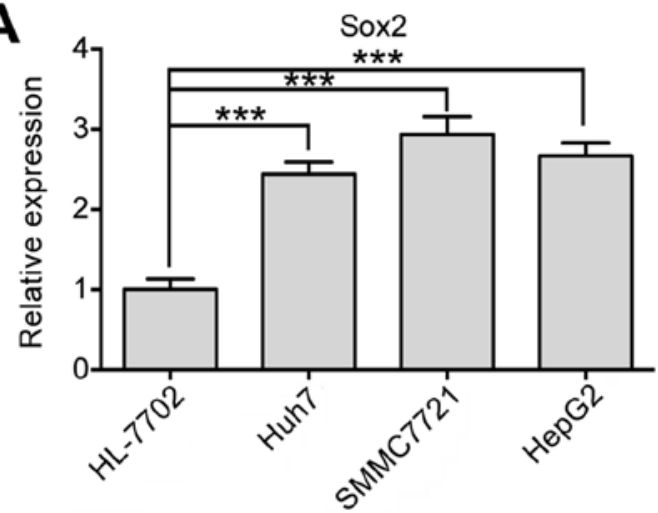

B
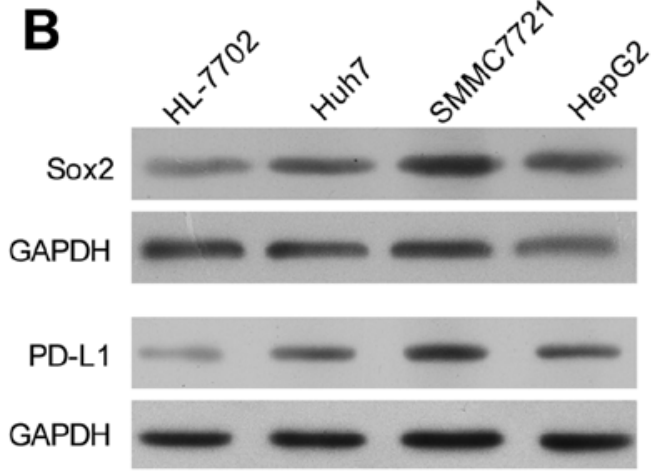

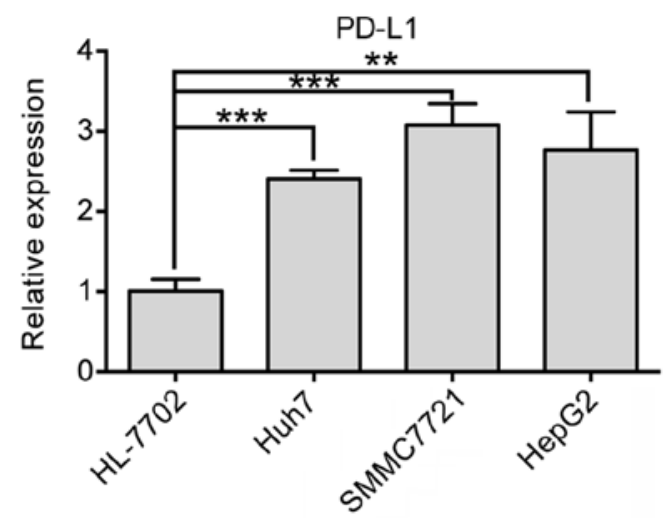

C

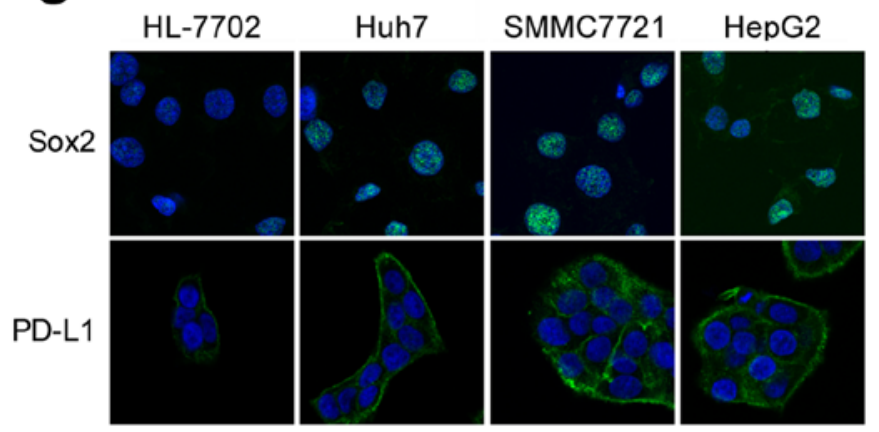

Figure 2. Sox 2 and PD-L1 expression are significantly higher in hepatocellular carcinoma cell line than in normal liver cell line. (A) RT-qPCR show the expression level of Sox2 (left panel) and PD-L1 (right panel) are significantly higher in hepatocellular carcinoma cell line, i.e, HepG2, SMMC7721 and Huh7 than that in normal liver cell line. Western blotting (B) and immunofluorescence (C) show the protein level of Sox 2 and PD-L1 are higher in three carcinoma cell lines than that in normal liver cell line. ${ }^{* *} \mathrm{p}<0.01 ;{ }^{* * *} \mathrm{p}<0.001$.

protein level of both proteins through western blot assays. As shown in Fig. 2B, the protein level of Sox 2 and PD-L1 were higher in HCC cell lines than that in the normal liver cell line. Moreover, we performed immunofluorescence assays to visualize the expression of Sox 2 and PD-L1 globally. As illustrated in Fig. 2C, both Sox 2 and PD-L1, even though they localized in the different cellular component, showed higher fluorescence intensity in HCC cell lines than in normal liver cell line. These data are consistent with the aforementioned results shown in Fig. 1 and provide strong evidence that Sox 2 and PD-L1 are both highly expressed in HCC.

Knockdown of Sox2 represses the proliferation growth and induces apoptosis of HCC cells. The finding that Sox 2 is highly expressed in HCC cells prompted us to elucidate the biological function of Sox 2 in HCC. We utilized an siRNA approach to knock down Sox 2 expression in Sox 2 highly expressed HCC cell lines (HepG2 and SMMC7721), and compared the cell proliferation ability and the status of cellular apoptosis before and after Sox 2 knockdown. The reduction of Sox 2 expression in Sox2-specific siRNA transfected cells was confirmed by qRT-PCR and western blot assays (Fig. 3A). The effect of Sox 2 depletion on cell proliferation was evaluated through using CCK-8 and EdU assays. We observed a significant decrease in proliferation after transfection of siSox2 (Fig. 3B and C) in both HepG2 and SMMC7721 cells. Sox 2 knockdown also led to apoptosis of HCC cells, based on the observations of the percentage of the Annexin V-positive and PI-negative cells (increased from 8.3 to $31.0 \%$ and 10.0 to $29.4 \%$, in HepG2 and SMMC7221 after Sox 2 knockdown, respectively) (Fig. 3D). Taken together, these data indicate that the Sox 2 oncogene is required for the proliferation and growth of HCC cells.

Sox2 transactivates $P D-L 1$ through the -757 region of the $P D-L 1$ promoter. Next, we asked whether Sox 2 regulates the expression of PD-L1. Through RT-qPCR and western blotting, we found that the expression level of PD-L1 was significantly decreased after Sox 2 knockdown (Fig. 4A), which indicated that the expression of PD-L1 might be regulated by Sox2. One important problem that remains to be clarified is whether Sox2 directly target the PD-L1 promoter in HCC cells. To address this question, we first investigated the transcription factor binding sites within the promoter region of PD-L1 using the TRANSFAC database (31). Of note, we found a consensus Sox 2 motif upstream of the transcription start site (TSS) at position -757 (Fig. 5A). Then we performed in vitro EMSA and in vivo ChIP-qPCR assay on Sox 2 motif containing promoter regions. As demonstrated in Fig. 5B, when a 22 bp probe contained the Sox 2 motif was incubated with nuclear extracts from HepG2, a specific DNA-protein complex was observed (lane 2, GS-Sox2) and was supershifted by the anti-Sox 2 antibody (lane 5, SS-Sox2). This indicated that Sox 2 bound to the promoter regions of PD-L1 directly through the Sox 2 motif. This was further verified by ChIP-qPCR assays in HepG2 cells using primers marked in Fig. 5A and antibodies against Sox 2 .

To further investigate whether Sox 2 transactivates the regulatory region of PD-L1 through the Sox 2 binding site identified 
A

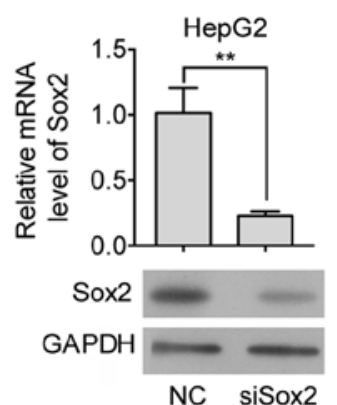

C
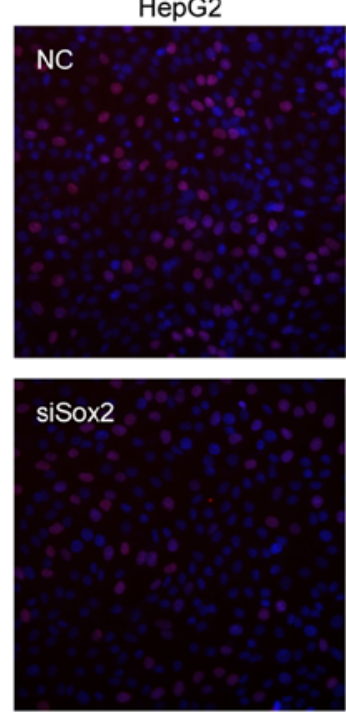

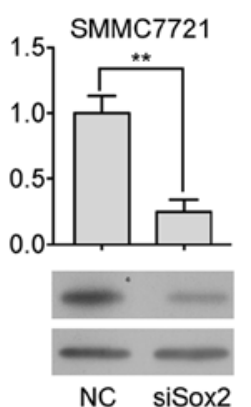

SMMC7721
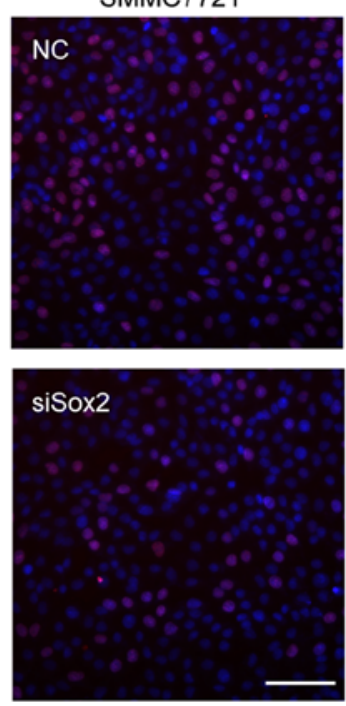
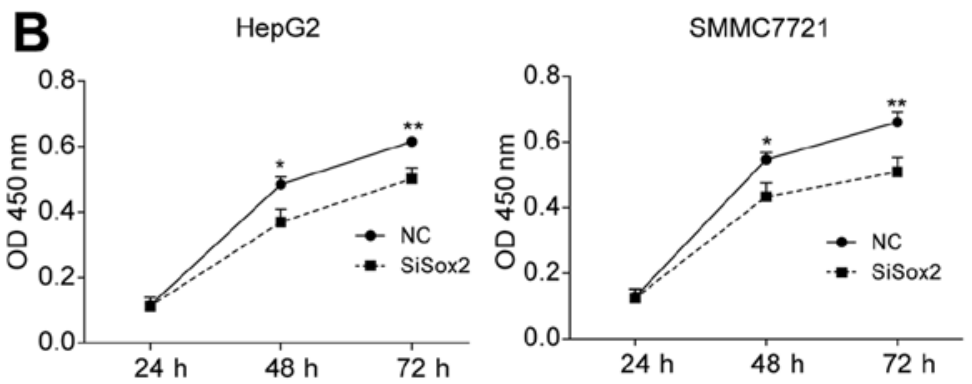

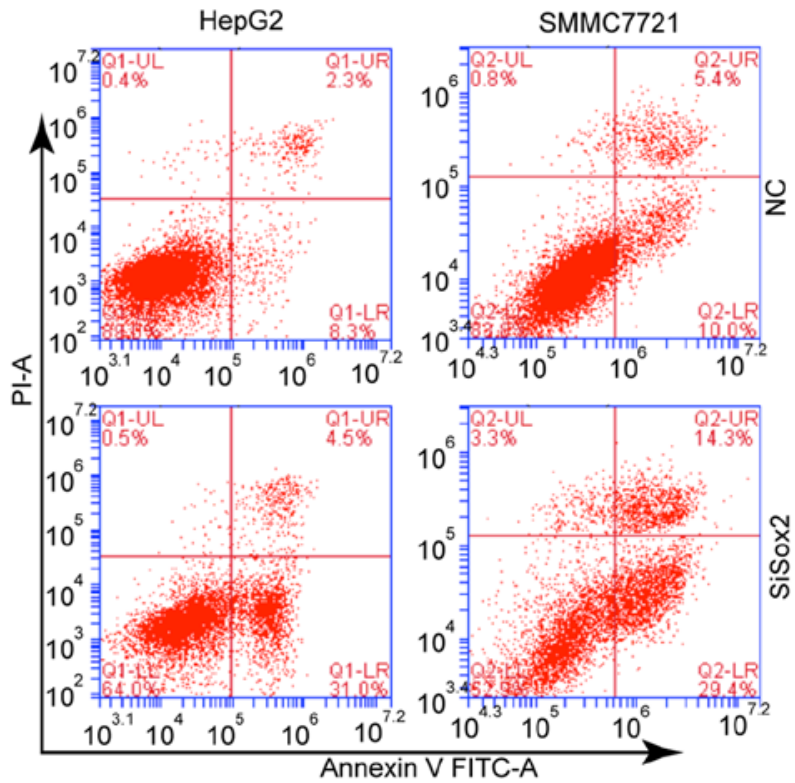

Figure 3. Knockdown of Sox 2 repress the growth and induce the apoptosis of HCC cells. (A) RT-qPCR and western blot analysis of Sox 2 expression. HepG2 and SMMC7721 cells were harvest after $72 \mathrm{~h}$ of after NC or siSox 2 transfection. (B) CCK-8 assays showed that Sox 2 knockdown repress the growth of HCC cell lines. The CCK-8 assays were performed on HepG2 and SMMC7721 cells after 24, 48 and $72 \mathrm{~h}$ after Sox 2 knockdown. Error bars represent the SD of triplicate measurements. (C) The BrdU assays showed that Sox 2 knockdown repress the proliferation of HCC cells. (D) Sox 2 knockdown induced apoptosis of HCC cell lines. Scar bar: $100 \mu \mathrm{m} .{ }^{*} \mathrm{p}<0.05 ;{ }^{* *} \mathrm{p}<0.01$.
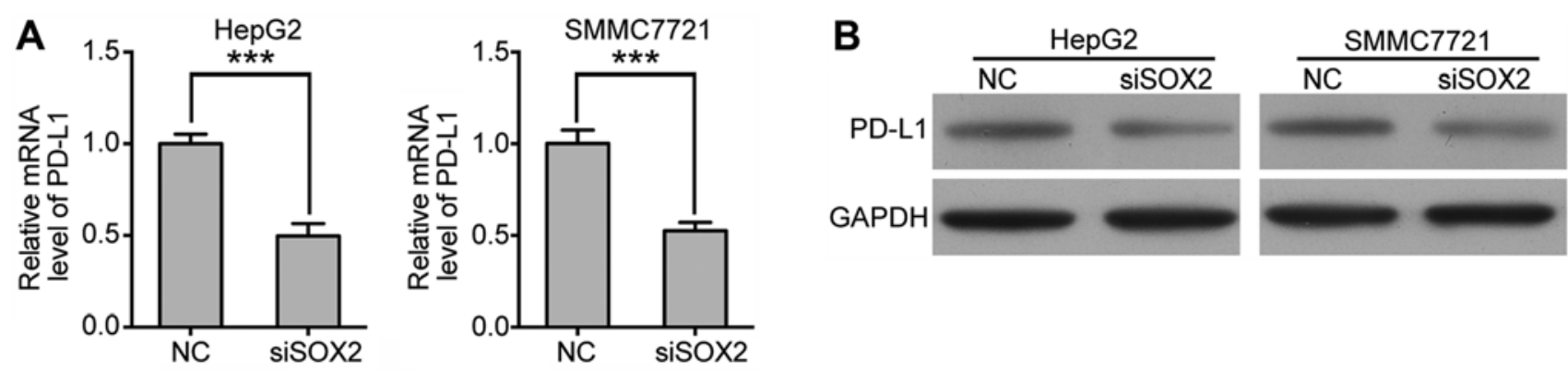

Figure 4. Sox 2 knockdown significantly decreases the expression level of PD-L1. The pd-11 expression is repressed in the Sox 2 knockdown cells (HepG2 and SMMC7721). The mRNA (A) and protein levels (B) of PD-L1 were detected by RT-qPCR (A) and western blotting (B). Error bars represent the SD of triplicate measurements. ${ }^{* * * *} \mathrm{p}<0.001$.

above, we performed luciferase reporter assays in HEK-293T cells. We cloned the promoter region of PD-L1 that contained Sox 2 motif and TSS into a luciferase reporter plasmid, which was defined as pGL3-PD-L1. We also constructed a pGL3PD-L1-mutant plasmid, in which the core sequence of Sox2 motif around $-757 \mathrm{bp}$ was mutated from CCATCGTC to TGCAGACG. As shown in Fig. 5D, co-expression of Sox2 resulted in significant activation of the PD-L1 promoter region. In contrast, the transactivation ability of Sox 2 on the
PD-L1 promoter region was significantly reduced as observed in the PD-L1 mutant promoter. Collectively, the data indicate that Sox 2 could transactivate the PD-L1 promoter through the Sox 2 motif located at the -757 bp upstream of TSS.

\section{Discussion}

In the last few years, numerous studies have been carried out to uncover the molecular markers during the development of 
A

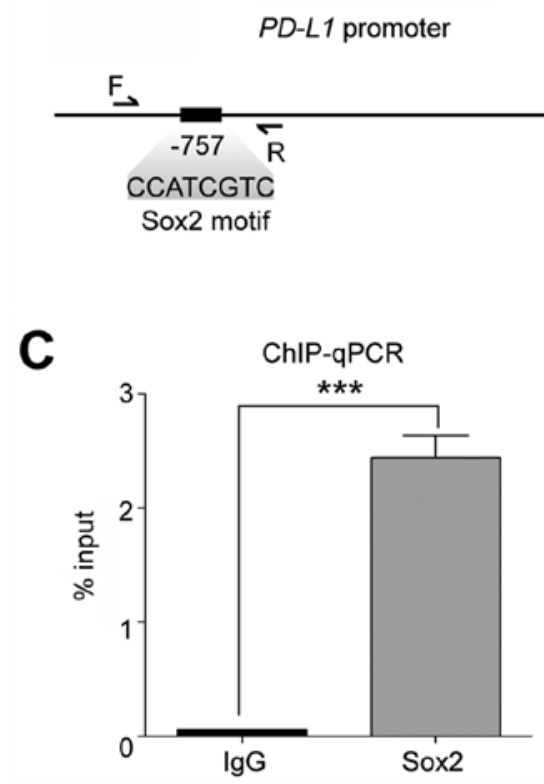

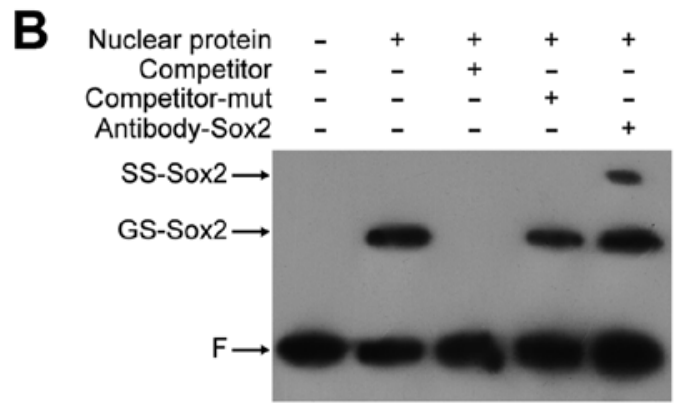

D

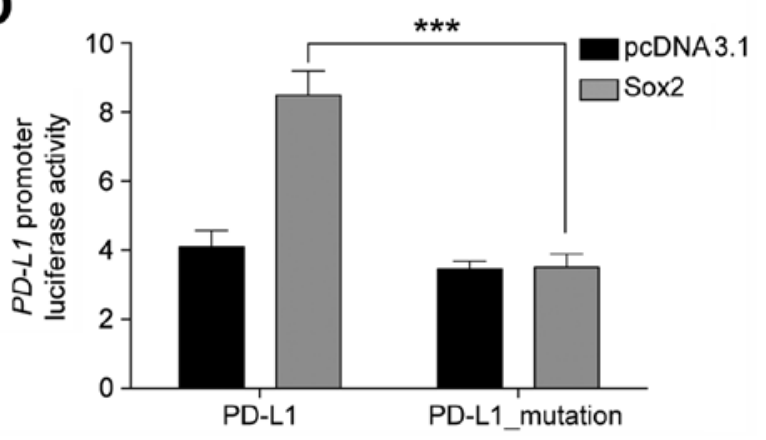

Figure 5. Sox 2 activates the expression of PD-L1 through directly binding and activation of the promoter region of PD-L1. (A) Schematic plot represent the promoter region of PDL-L1. Sox 2 binding sites are defined using TRANSFAC. Arrows show the primer location of ChIP-qPCR. (B) Demonstration of the binding of Sox 2 and DNA probe containing Sox 2 motif by EMSA assays. A Sox 2 motif-containing probe from the PD-L1 promoter was used. SS-Sox2, super-shifted Sox2-DNA complex with the anti-Sox2 antibody; GS-Sox2, gel-shifted complex formed with Sox2; F, unbound free probe. Nuclear protein was extracted from HepG2 cells. (C) Sox 2 bound to the promoter region of PD-L1. ChIP-qPCR was performed in HepG2 cells. Error bars represent the SD of triplicate measurements. (D) Sox 2 activates PD-L1 promoter activity through the DNA binding motif. Error bars represent the SD of triplicate measurements. ${ }^{* * *} \mathrm{p}<0.001$.

HCC $(32,33)$; however, the actual mechanisms of how these molecular markers lead to malignant transformation are not yet well understood. Our data indicate that both Sox 2 and PD-L1 are highly expressed in hepatocellular carcinoma (HCC). Functional analysis shows the expression of Sox 2 is closely related to the proliferation and growth of HCC cells. We also found that PD-L1, a well-qualified immunotherapeutic target in various solid tumors, is transactivated by Sox 2 . These findings provide novel insight into the function and the interplay between Sox 2 and PD-L1 in HCC, and significantly enrich our understanding of the role of Sox 2 in HCC malignancy.

Sox 2 is a potential biomarker for HCC prognosis $(18,34)$. Previous research including 75 patient samples concluded that high expression of Sox 2 in HCC tissues was positively correlated with malignancy and poor survival (34). In our study, we show that the expression of Sox 2 is significantly higher in HCC tissue from patient samples and HCC cell lines. This observation is consistent with several previous reports. Moreover, we ivestigated the function of Sox 2 in HCC and found that Sox 2 is needed for keeping the proliferation ability and prevent HCC cells from apoptosis. Our study not only provides direct evidence (in tissue samples and cell lines) to support the expression pattern of Sox 2 but also further explores the Sox 2 function. In summary, our finding supports the fact that Sox 2 may function as an oncogene in solid tumors especially in HCC.

PD-L1 is an immunotherapeutic target in various solid tumors, such as NSCLC, bladder cancer and renal cell carcinoma $(20,21,23,26)$. We demonstrate here that the expression level of PD-L1 is higher in HCC tissues/cells than that in normal tissues/cells. Recent attention to the role of PD-L1 in the tumor progress of HCC has provided worthy insights into the mechanism of HCC development and associated therapeutic approaches. For example, the PD-L1 expression in HCC is associated with the tumor malignancy and the postoperative recurrence risk $(22,35)$. Accordingly, we tentatively propose that PD-L1 might represent a potential target for HCC immunotherapy.

We observed that the cellular location of PD-L1 and Sox2 are different. In most instances, Sox 2 localized only in the nucleus (Fig. 2C). The distribution pattern of Sox 2 is due to Sox 2 function as a transcriptional factor, and it functions through binding to the promoter regions of its target gene in the nucleus $(10,36,37)$. The location pattern we found here is different from a previous report, which shows that Sox2 locates not only in the nucleus, but also in the cytoplasm of HCC tissue and cell lines, this might be caused by the different method we used for immunofluorescence. On the other hand, as confirmed (38), PD-L1 is located in the cytoplasm and on the cell membrane of tumor cells and immune cells, we observed that PD-L1 indeed localized on the cell membrane and partly in the cytoplasm. It seems that the fluorescence single on the cell membrane is slightly stronger than that in the cytoplasm.

In summary, our findings not only reveal the relationship between Sox 2 and PD-L1, but also enrich our understanding of the potential role of Sox 2 in HCC malignancy.

\section{References}

1. Farazi PA and DePinho RA: Hepatocellular carcinoma pathogenesis: From genes to environment. Nat Rev Cancer 6: 674-687, 2006.

2. Simonetti RG, Liberati A, Angiolini C and Pagliaro L: Treatment of hepatocellular carcinoma: A systematic review of randomized controlled trials. Ann Oncol 8: 117-136, 1997. 
3. Mas VR, Fisher RA, Archer KJ, Yanek KC, Williams B, Dumur CI and Maluf DG: Genes associated with progression and recurrence of hepatocellular carcinoma in hepatitis C patients waiting and undergoing liver transplantation: Preliminary results. Transplantation 83: 973-981, 2007.

4. Moradpour D and Blum HE: Pathogenesis of hepatocellular carcinoma. Eur J Gastroenterol Hepatol 17: 477-483, 2005.

5. Nam SW, Park JY, Ramasamy A, Shevade S, Islam A, Long PM, Park CK, Park SE, Kim SY, Lee SH, et al: Molecular changes from dysplastic nodule to hepatocellular carcinoma through gene expression profiling. Hepatology 42: 809-818, 2005.

6. Poon TC, Wong N, Lai PB, Rattray M, Johnson PJ and Sung JJ: A tumor progression model for hepatocellular carcinoma: Bioinformatic analysis of genomic data. Gastroenterology 131: 1262-1270, 2006.

7. Teufel A, Staib F, Kanzler S, Weinmann A, Schulze-Bergkamen H and Galle PR: Genetics of hepatocellular carcinoma. World J Gastroenterol 13: 2271-2282, 2007.

8. Schafer DF and Sorrell MF: Hepatocellular carcinoma. Lancet 353: 1253-1257, 1999.

9. Mas VR, Maluf DG, Archer KJ, Yanek K, Kong X, Kulik L, Freise CE, Olthoff KM, Ghobrial RM, McIver P, et al: Genes involved in viral carcinogenesis and tumor initiation in hepatitis $C$ virus-induced hepatocellular carcinoma. Mol Med 15: 85-94, 2009.

10. Rodda DJ, Chew JL, Lim LH, Loh YH, Wang B, Ng HH and Robson P: Transcriptional regulation of nanog by OCT4 and SOX2. J Biol Chem 280: 24731-24737, 2005.

11. Bass AJ, Watanabe H, Mermel CH, Yu S, Perner S, Verhaak RG, Kim SY, Wardwell L, Tamayo P, Gat-Viks I, et al: SOX2 is an amplified lineage-survival oncogene in lung and esophageal squamous cell carcinomas. Nat Genet 41: 1238-1242, 2009.

12. Chen Y, Shi L, Zhang L, Li R, Liang J, Yu W, Sun L, Yang X, Wang Y, Zhang Y, et al: The molecular mechanism governing the oncogenic potential of SOX2 in breast cancer. J Biol Chem 283: 17969-17978, 2008

13. Hussenet T, Dali S, Exinger J, Monga B, Jost B, Dembelé D, Martinet N, Thibault C, Huelsken J, Brambilla E, et al: SOX2 is an oncogene activated by recurrent 3q26.3 amplifications in human lung squamous cell carcinomas. PLoS One 5: e8960, 2010.

14. Hussenet $\mathrm{T}$ and du Manoir S: SOX2 in squamous cell carcinoma Amplifying a pleiotropic oncogene along carcinogenesis. Cell Cycle 9: 1480-1486, 2010

15. Justilien V, Walsh MP, Ali SA, Thompson EA, Murray NR and Fields AP: The PRKCI and SOX2 oncogenes are coamplified and cooperate to activate Hedgehog signaling in lung squamous cell carcinoma. Cancer Cell 25: 139-151, 2014.

16. Schoenhals M, Kassambara A, De Vos J, Hose D, Moreaux J and Klein B: Embryonic stem cell markers expression in cancers. Biochem Biophys Res Commun 383: 157-162, 2009.

17. Huang P, Qiu J, Li B, Hong J, Lu C, Wang L, Wang J, Hu Y Jia W and Yuan Y: Role of Sox 2 and Oct4 in predicting survival of hepatocellular carcinoma patients after hepatectomy. Clin Biochem 44: 582-589, 2011.

18. Sun C, Sun L, Li Y, Kang X, Zhang S and Liu Y: Sox2 expression predicts poor survival of hepatocellular carcinoma patients and it promotes liver cancer cell invasion by activating Slug. Med Oncol 30: 503, 2013.

19. Zhao C, Li Y, Zhang M, Yang Y and Chang L: miR-126 inhibits cell proliferation and induces cell apoptosis of hepatocellular carcinoma cells partially by targeting Sox 2 . Hum Cell 28: 91-99, 2015.

20. Blank C, Kuball J, Voelkl S, Wiendl H, Becker B, Walter B, Majdic O, Gajewski TF, Theobald M, Andreesen R, et al: Blockade of PD-L1 (B7-H1) augments human tumor-specific T cell responses in vitro. Int J Cancer 119: 317-327, 2006.

21. Herbst RS, Soria JC, Kowanetz M, Fine GD, Hamid O, Gordon MS, Sosman JA, McDermott DF, Powderly JD, Gettinger SN, et al Predictive correlates of response to the anti-PD-L1 antibody MPDL3280A in cancer patients. Nature 515: 563-567, 2014.

22. Zeng Z, Shi F, Zhou L, Zhang MN, Chen Y, Chang XJ, Lu YY, Bai WL, Qu JH, Wang CP, et al: Upregulation of circulating PD-L1/PD-1 is associated with poor post-cryoablation prognosis in patients with HBV-related hepatocellular carcinoma. PLoS One 6: e23621, 2011.
23. Nomi T, Sho M, Akahori T, Hamada K, Kubo A, Kanehiro H, Nakamura S, Enomoto K, Yagita H, Azuma M, et al: Clinical significance and therapeutic potential of the programmed death-1 ligand/programmed death-1 pathway in human pancreatic cancer. Clin Cancer Res 13: 2151-2157, 2007.

24. Butte MJ, Keir ME, Phamduy TB, Sharpe AH and Freeman GJ: Programmed death-1 ligand 1 interacts specifically with the B7-1 costimulatory molecule to inhibit $\mathrm{T}$ cell responses. Immunity 27 : 111-122, 2007.

25. Park JJ, Omiya R, Matsumura Y, Sakoda Y, Kuramasu A, Augustine MM, Yao S, Tsushima F, Narazaki H, Anand S, et al: B7-H1/CD80 interaction is required for the induction and maintenance of peripheral T-cell tolerance. Blood 116: 1291-1298, 2010.

26. Paterson AM, Brown KE, Keir ME, Vanguri VK, Riella LV, Chandraker A, Sayegh MH, Blazar BR, Freeman GJ and Sharpe AH: The programmed death-1 ligand 1:B7-1 pathway restrains diabetogenic effector T cells in vivo. J Immunol 187: 1097-1105, 2011.

27. Motzer RJ, Rini BI, McDermott DF, Redman BG, Kuzel TM, Harrison MR, Vaishampayan UN, Drabkin HA, George S, Logan TF, et al: Nivolumab for metastatic renal cell carcinoma: results of a randomized phase II trial. J Clin Oncol 33: 1430-1437, 2015.

28. Robert C, Ribas A, Wolchok JD, Hodi FS, Hamid O, Kefford R, Weber JS, Joshua AM, Hwu WJ, Gangadhar TC, et al: Antiprogrammed-death-receptor-1 treatment with pembrolizumab in ipilimumab-refractory advanced melanoma: A randomised dose-comparison cohort of a phase 1 trial. Lancet 384: 1109-1117, 2014.

29. Gao Q, Wang XY, Qiu SJ, Yamato I, Sho M, Nakajima Y, Zhou J, Li BZ, Shi YH, Xiao YS, et al: Overexpression of PD-L1 significantly associates with tumor aggressiveness and postoperative recurrence in human hepatocellular carcinoma. Clin Cancer Res 15: $971-979,2009$

30. Li Y, Wang H, Wang X, Jin W, Tan Y, Fang H, Chen S, Chen Z and Wang K: Genome-wide studies identify a novel interplay between AML1 and AML1/ETO in $\mathrm{t}(8 ; 21)$ acute myeloid leukemia. Blood 127: 233-242, 2016

31. Matys V, Kel-Margoulis OV, Fricke E, Liebich I, Land S, BarreDirrie A, Reuter I, Chekmenev D, Krull M, Hornischer K, et al: TRANSFAC and its module TRANSCompel: Transcriptional gene regulation in eukaryotes. Nucleic Acids Res 34: D108-D110, 2006.

32. Lin R, Maeda S, Liu C, Karin M and Edgington TS: A large noncoding RNA is a marker for murine hepatocellular carcinomas and a spectrum of human carcinomas. Oncogene 26: 851-858, 2007.

33. Sakamoto M: Early HCC: Diagnosis and molecular markers. J Gastroenterol 44 (Suppl 19): 108-111, 2009.

34. Xu X, Liu RF, Zhang X, Huang LY, Chen F, Fei QL and Han ZG: DLK1 as a potential target against cancer stem/progenitor cells of hepatocellular carcinoma. Mol Cancer Ther 11: 629-638, 2012.

35. Shi F, Shi M, Zeng Z, Qi RZ, Liu ZW, Zhang JY, Yang YP, Tien P and Wang FS: PD-1 and PD-L1 upregulation promotes CD8(+) T-cell apoptosis and postoperative recurrence in hepatocellular carcinoma patients. Int J Cancer 128: 887-896, 2011.

36. Pan X, Cang X, Dan S, Li J, Cheng J, Kang B, Duan X, Shen B and Wang YJ: Site-specific disruption of the Oct4/Sox2 protein interaction reveals coordinated mesendodermal differentiation and the epithelial-mesenchymal transition. J Biol Chem 291: 18353-18369, 2016.

37. Scaffidi P and Bianchi ME: Spatially precise DNA bending is an essential activity of the sox 2 transcription factor. J Biol Chem 276: 47296-47302, 2001.

38. Wu C, Zhu Y, Jiang J, Zhao J, Zhang XG and Xu N: Immunohistochemical localization of programmed death-1 ligand-1 (PD-L1) in gastric carcinoma and its clinical significance. Acta Histochem 108: 19-24, 2006. 